\title{
Weighted Interaction Force Estimation for Abnormality Detection in Crowd Scenes
}

\author{
Xiaobin Zhu, Jing Liu, Jinqiao Wang, Wei Fu, Hanqing Lu \\ National Laboratory of Pattern Recognition, Institute of Automation, \\ Chinese Academy of Sciences, Beijing 100190, China \\ $\{x b z h u$, jliu, jqwang, wfu, luhq\}@nlpr.ia.ac.cn
}

\begin{abstract}
In this paper, we propose a weighted interaction force estimation in the social force model(SFM)-based framework, in which the properties of surrounding individuals in terms of motion consistence, distance apart, and angle-of-view along moving directions are fully utilized in order to more precisely discriminate normal or abnormal behaviors of crowd. To avoid the challenges in object tracking in crowded videos, we first perform particle advection to capture the continuity of crowd flow and use these moving particles as individuals for the interaction force estimation. For a more reasonable interaction force estimation, we jointly consider the properties of surrounding individuals, assuming that the individuals with consistent motion (as a particle group) and the ones out of the angle-of-view have no influence on each other, besides the farther apart ones have weaker influence. In particular, particle groups are clustered by spectral clustering algorithm, in which a novel and high discriminative gait feature in frequency domain, combined with spatial and motion feature, is used. The estimated interaction forces are mapped to image span to form force flow, from which bag-of-word features are extracted. Sparse Topical Coding (STC) model is used to find abnormal events. Experiments conducted on three datasets demonstrate the promising performance of our work against other related ones.
\end{abstract}

\section{Introduction}

One of the most challenging tasks in computer vision is behavior analysis in crowd scenes, because it requires monitoring an excessive number of individuals and their activities, and retaining structural information regarding the entire scene. A central task of crowd behavior analysis involves detecting or even predicting abnormal behaviors, i.e., finding new patterns in data that do not conform to the expected case. Figure. 1 shows two examples of abnormal events: (a) running away in panic, and (b) fighting in street. Abnormality detection plays an important role in video surveillance context.

Abnormality detection in crowd scenes is thoroughly studied by the computer vision communities, where some well established models have been developed. Recently, social force model (SFM) is widely used in abnormality detection and shows us satisfied performance. The SFM was originally introduced by Helbing 


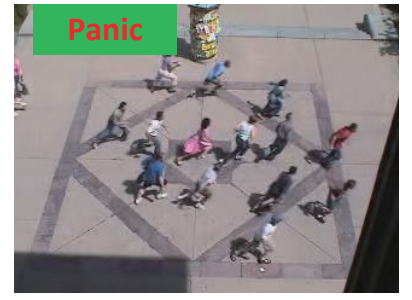

(a)

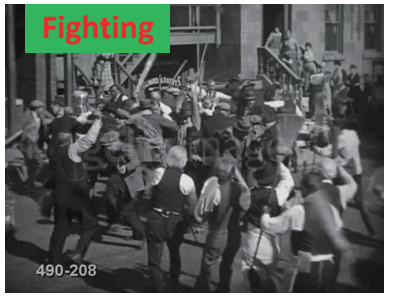

(b)

Fig. 1. Abnormal event examples. (a) People are running away in panic; (b) People are fighting.

et al. [1] to investigate the pedestrian movement dynamics. It provides a mathematical formalization to describe the movement of each individual in a crowd on the basis of its interaction with the surroundings. Thus, a reasonable consideration of different influences from surrounding individuals is important to obtain an effective estimation of the interaction force. This is also our focus in this paper.

To address the above issue, we propose a new approach for interaction force estimation in the SFM-based framework. In our approach, the properties of surrounding individuals in terms of motion consistence, distance apart, and angle-ofview along moving directions are fully utilized during interaction force estimating procedure. The framework of proposed approach is summarized in Figure. 2. First, moving particles are extracted by particle advection using algorithm [2], and treated as individuals in the interaction force estimation procedure. Second, interaction force among particles is estimated by the SFM, in which the above mentioned properties of surrounding individuals are jointly considered. To obtain the particle groups with consist motion patterns, we conduct spectral clustering algorithm by exploring a kind of combined feature representation, including discriminative gait feature in frequency domain, motion coherency feature and spatial proximity feature. In our work, each group is considered as a set of particles with similar motion pattern in local nearby region. As an ideal solution, each group corresponds to a separated functional part or subpart of human body, e.g., head, hand. We assume that particles within the same group have no influence on each other, i.e., no repulsive interaction force. This is reasonable. Besides, the magnitude of the interaction force should be influenced by the angle-of-view along particles' moving directions. That is, given a particle, any particles lying in its forward view should cause influence on it, while the ones out of its view (behind the given particle) should have no influence. The distance among particles is also considered in the interaction force estimation in our work. In the following, the estimated interaction force corresponding to each particle will be mapped to image frames to obtain force flow across video frames. With the resulting force flow, bag-of-word features are extracted, and Sparse Topical Coding (STC) [3] is used to classify normal and abnormal events.

The rest of the paper is organized as follows. Section 2 overviews the related works. In Section 3, we introduce our SFM-based abnormality detection 


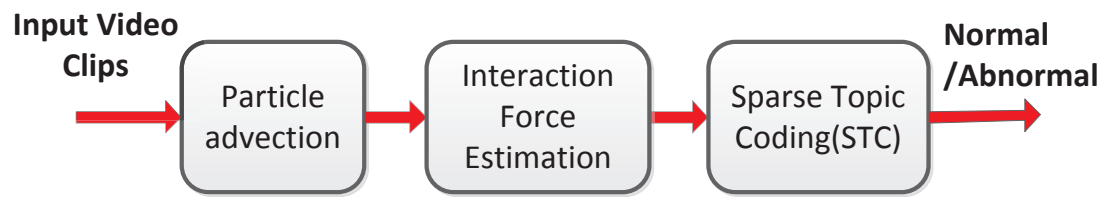

Fig. 2. The framework of the proposed approach.

approach. In Section 4, we elaborate the estimation of the interaction force. The experimental evaluation is given in Section 5, and we draw conclusion in Section 6 .

\section{Related Work}

Abnormality detection is an active area of research on its own. Various approaches have been proposed, for both the crowd and non-crowd scenes. In the following, we will focus on the case of crowd scenes. The works relevant to the crowd scenes can be broadly divided into two categories, according to the type of scene representation adopted. One category is object-based approach, which considers the group as a collection of individuals [4][5][6]. To understand crowd behavior, segmentation, detection, or tracking should be performed in advance. In simple scene, such approaches can achieve good performance. However, in crowd scenes, object occlusions can severely affect the accuracy of segmentation or tracking, which can heavily degrade the performance of detection. Additionally, the computational cost will also be tremendous when various objects exist. The other category adopts motion representations regarding to the whole scene that avoids tracking. In this work, we focus on addressing this issue.

The most popular approaches are based on optical flow, or spatio-temporal gradients $[7][8][9][10][11][12][13]$, where the majority are based on particle advection algorithms. Particles are image points distributed on a frame and advected according to underlying motion. The first work adopting particle advection algorithm for crowd behavior analysis was introduced in [11]. Here, the particle flow is computed by moving a grid of particles using the fourth-order Runge-KuttaFehlberg algorithm along with the bilinear interpolation of the optical flow field. This method was further extended in [10] using chaotic invariants capable of analyzing both coherent and incoherent scenes. In [12], sreaklines are introduced and integrated with a particle advection scheme capable of incorporating the spatial change in the particle flow. In [7], the social force model (SFM) [1] was exploited using a simplified social force model to detect abnormal events. After putting a fixed grid of particles on each frame, the SFM is used to estimate the interaction force under the belief that it contains significant information for describing (abnormal) crowd behavior. After estimating the so-called force flow, a bag of words method and Latent Dirichlet Allocation (LDA) [14] are employed for abnormality detection, while localizing the abnormal areas as those with the highest force 
magnitude. In [13], Particle Swarm Optimization (PSO) method was introduced as a robust algorithm for optimizing the interaction force computed using SFM. However, the threshold method used for detecting anomaly is too arbitrary and scene dependent. In above algorithms, the properties of surrounding individuals in terms of motion consistence, distance apart, and angle-of-view, are not well taken into consideration, during interaction force estimation.

In addition to the above mentioned particle advection schemes, there also exist a few model-based approaches tackling the same problem. Kratz and Nishino [8] proposed a spatial-temporal model, in which a distribution-based Hidden Markov Model (HMM) is used to describe the motion transitions in the local video regions. However, this method may work only for one kind of normal behavior type in the scene. Yang et al. [9] proposed a novel algorithm for abnormal event detection based on the sparse reconstruction cost for multi-level histogram of optical flows. However, the multi-level histogram of optical flow individually cannot well reflect the dynamics of crowd individually.

\section{SFM-based Abnormality Detection}

The framework of our approach is summarized in Figure. 2. For obtaining moving particles, we conduct particle advection using algorithm in [2] in advance. Then interaction force is estimated using SFM. Features are represented by the interaction force with a bag-of-word scheme. Finally, STC [3] is used to classify normal and abnormal events.

\subsection{Particle Advection}

In crowd, tracking of objects can cause extensive clutter and occlusions. To avoid tracking of objects, in earlier works [7][11], the particle advection is carried out by placing a rectangular grid of particles over each video frame. Then, the velocity of each particle is calculated using fourth-order Runge-Kutta Fehlberg algorithm [15] along with the bilinear interpolation of the optical flow field. A drawback of this approach is the elements of the crowd may move with unpredictable trajectories that will result in an unstructured flow. To avoid this drawback, we conduct particle advection using algorithm in [2]. The resulting moving particles, which belong to different trajectories, have a more strong ability to model complex motion and occlusion. Figure. 3(a) illustrates an example of particle advection.

\subsection{Social Force Model}

In the following, we introduce SFM for pedestrian motion dynamics by considering the individual (particles) motivations and environmental constraints. Each individual $i$ is driven by a force $F_{i}$. The force is not real physical force, but rather a measure of the individual motivation for acting in specific ways. The SFM can be written as:

$$
m_{i} \frac{d V_{i}}{d t}=F_{i}=F_{d}+F_{i n t}
$$




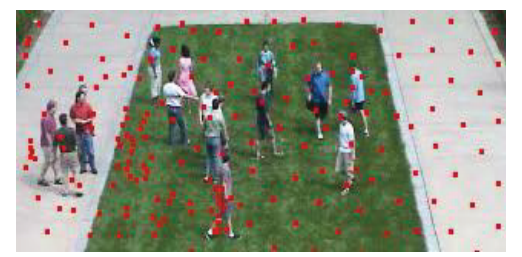

(a)

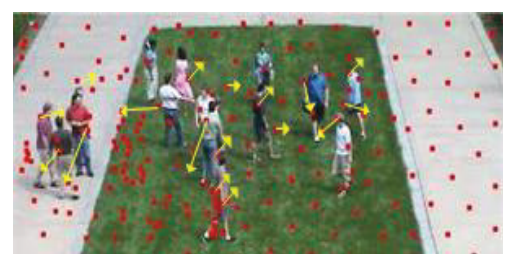

(b)

Fig. 3. An example of particle advection and interaction force. (a) Moving particles belong to their trajectories in a particular frame. (b) The computed interaction force of corresponding particles, the longer arrow means a higher value.

where $m_{i}$ denotes the mass of the individual, $V_{i}$ indicates its actual velocity, $F_{d}$ is the desired movement force, and $F_{i n t}$ indicates the interaction force.

The desired movement force represents the individual's desire to move towards its destination. The idea is that a pedestrian (individual), in unhindered, will move towards its target at an initial desired speed. The desired movement force is a velocity dependent force given by:

$$
F_{d}=\frac{1}{\tau}\left(V_{i}^{d}-V_{i}\right)
$$

where $V_{i}^{d}$ is the desired speed, he parameter $\tau$ is the relaxation time and scales the force, determining how fast a individual changes velocity and returns to its desired velocity after having been walking slower because of obstacles etc. The relaxation time is a model parameter that in principle can vary for each individual. However, from [16] it is clear that in practice, $\tau$ is the same for all individuals.

Due to the influence of surroundings, in this model, personal desired movement force $V_{i}^{d}$ is replaced with

$$
V_{i}^{p}=\left(1-\eta_{i}\right) V_{i}^{d}+\eta_{i}\left\langle V_{i}^{a v g}\right\rangle
$$

where $\eta_{i}$ is the panic weight parameter and $\left\langle V_{i}^{a v g}\right\rangle$ is the average velocity of the neighboring individuals. The individual $i$ exhibits individualistic behavior as $\eta_{i} \rightarrow 0$, and herding behaviors as $\eta_{i} \rightarrow 1$.

In this paper, we focus our attention on estimating the interaction force $F_{\text {int }}$ between individuals as a single quantity. And the detailed estimation of interaction force, considering influences of motion consistence, distance apart, and angle-of-view, will be elaborated in Section 4. Overall, the interaction force estimation in SFM can be summarized as

$$
F_{\text {int }}=m_{i} \frac{d V_{i}}{d t}-\frac{1}{\tau}\left(V_{i}^{p}-V_{i}\right)
$$

\subsection{Global Abnormality Detection}

The interaction forces computed reflect the energy of individual with his surroundings. To model the patterns of interaction forces over time, we map the 


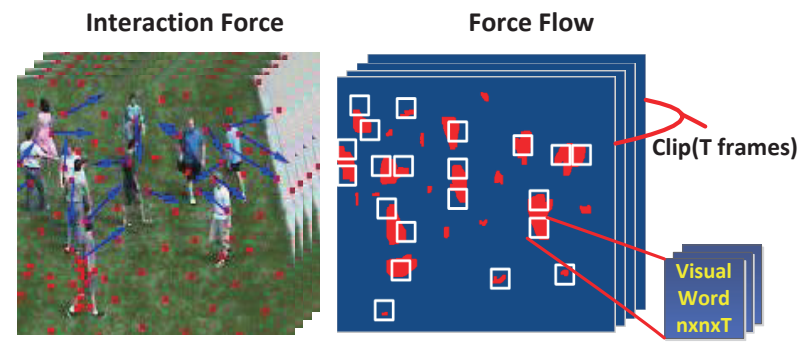

Fig. 4. The left picture shows the interaction force computed. The right picture shows the visual words are picked as $3 \mathrm{D}$ volumes of features from the force flow.

magnitude of the interaction force vectors to image plane such that there is a corresponding force vector for every pixel in the frame. As a result, for a stream of image frames $F(t)$, we construct a feature matrix of force flow of the same resolution with video frame. To use bag-of-word feature, we firstly segment the force flow into time length of $T$ frames. Then from each clip in force flow, we randomly pick visual words of size $n \times n \times T$ from locations, where interaction forces are not all zeros. Codebook is formed using K-means clustering. Figure. 4 illustrates the process of force flow computation and visual words extraction.

For a set of normal force flows of a group of similar scenes, we construct the flow word $\mathbf{w}=\left\{\mathbf{w}_{i}\right\}_{i=1}^{M}$, and use STC [3] to discover the distribution of $L$ topics for the normal crowd behavior. Different from LDA, by explicitly imposing a sparse constraint on the inferred representations in a non probabilistic formulation, Sparse Topical Coding has been proved to be an efficient approach to discover the semantic topical bases in collections of data, which is also suitable for motion patterns mining in videos [17]. And in our experiment, STC outperforms LDA with the same configuration. Using the STC, we approximate the bag of words model to maximize the objective function as

$$
f(\theta, \beta)=\sum_{i=1}^{M} p\left(\mathbf{w}_{i} \mid \beta, \theta\right)
$$

where $\beta$ is the dictionary of topics and $\theta$ is the document codes for each video clip. By using the model, we estimate the likelihood $\log p\left(\mathbf{w}_{i} \mid \beta, \theta\right)$ for every clip from the video sequence. And we classify the clips into normal or abnormal by a fixed threshold.

\section{Interaction Force Estimation}

In this section, we will elaborate the properties of surrounding individuals in terms of motion consistence, distance apart, and angle-of-view in interaction force estimation. 


\subsection{Particle Group Clustering}

The particle group information is important in interaction force estimation. The influence of moving particles in the same group should be zero [18]. We will use spectral clustering method to obtain the particle group information. In crowds, the motion of neighbor individuals tends to be very similar, because they are constrained by the motion of the crowd. Consequently, tracking or clustering methods [19][20] based on motion similarity and spatial proximity only have difficulty in discriminating between nearby individuals. Gait feature [21] have been shown to be an effective biometric cue in discriminating between individuals.

To obtain particle group information, we conduct graph clustering, using gait feature in frequency domain[21], combine with motion coherency and spatial proximity, on obtained trajectories in a video clip, which moving particles belonging to. As in [21], we also extract the periodic motion of an individual along with the vertical axis as the gait feature. To extract the gait feature for a given trajectory, we first fit a line to the trajectory through linear regression (depicted in Figure. 5), and then extract the periodic component $y_{p}(t)$ of the trajectory. Lets $Y_{i}$ represents the $i^{t h}$ component of $y_{p}(t)$, and $N$ is the total component number. We use the Fast Fourier Transform (FFT) with 64 points to find the amplitude spectra and phase of the trajectory.

$$
Y_{i}=\sum_{k=0}^{N-1} Y_{k} e^{-\frac{2 \pi i}{N} k}, i=0, \ldots, N-1
$$

The dissimilarity of a pair of trajectories is evaluated using Dynamic Time Warping $(D T W)$ algorithm based on amplitude spectra.
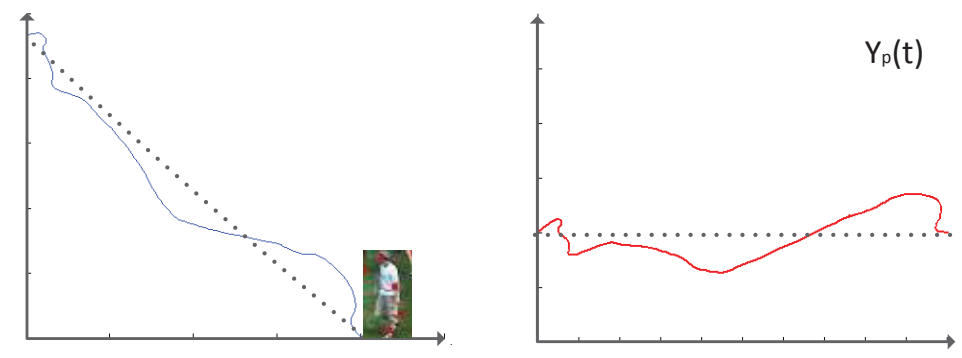

Fig. 5. Line fitting for removing linear motion component of feature trajectory(left image). Extracted periodic motion $y_{p}(t)$ (right image).

To perform clustering, we firstly construct a graph in each time window (video clip). Each node of the graph corresponds to a separate trajectory in clip, the edges connecting the nodes are identical to links in [2], which are obtained via Delaunay triangulation [22]. The edge connecting two nodes $i$ and $j$ is assigned a weight representing the dissimilarity between the corresponding trajectories based on three metrics: (1) gait feature, (2) spatial proximity, borrowed from 
[20], (3) motion difference, borrowed from [2]. With these weights at hand, the total edge weight is computed as the product of each individual weight.

$$
e^{i, j}=e_{g}^{i, j} e_{m}^{i, j} e_{p}^{i, j}
$$

where right hand side of Eq. 7 denotes the weight based on the gait feature $e_{g}^{i, j}$, coherency of motion $e_{m}^{i, j}$, and the spatial proximity $e_{p}^{i, j}$ respectively. The graph is then clustered into different particle groups.

\subsection{Computing the Interaction Force}

The desired movement force represents the individual's desire to move towards its destination. However, the individual action will be affected by surroundings. In Eq. $3, V_{i}^{d}$ is defined as the maximum speed of individual in the trajectory. $\left\langle V_{i}^{a v g}\right\rangle$ is the average velocity of surrounding individuals, considering the influence of particle group information, distance, and angle-of-view, and computed as:

$$
\left\langle V_{i}^{a v g}\right\rangle=\frac{1}{K} \sum_{j=0}^{K-1} f_{i j}(t) V_{j}
$$

where $K$ is the total number of surrounding moving particles in time $t$ for moving particle $i, V_{j}$ is the optical flow of moving particle $j$ in the coordinate $\left(x_{j}, y_{j}\right)$, $f_{i j}(t)$ is the influence factor of $j$ to $i$. The influence of surrounding individuals considering the distance, angle-of-view, particle group information, is given by:

$$
\begin{gathered}
f_{i j}(t)=\omega_{i j}^{d} \omega_{i j}^{\phi} \omega_{i j}^{g} \\
\omega_{i j}^{d}=-\exp \left(\frac{d_{i j}^{2}}{2 \sigma_{d}^{2}}\right) \\
\omega_{i j}^{\phi}= \begin{cases}1, & \phi_{i j}<\phi_{v i e w} \\
0, & \text { otherwise }\end{cases} \\
\omega_{i j}^{g}= \begin{cases}0, & i, j \in \text { same particle group } \\
1, & \text { otherwise }\end{cases}
\end{gathered}
$$

where $\sigma_{d}$ is the radius of influence. The closer of individual $j$, the higher impaction should be caused to individual $i$. As shown in Figure. 6, the particle P3 will have higher impaction on particle $P 1$ than $P 6$. $\phi_{\text {view }}$ is the angle-of-view, which is the angle displacement between the current moving direction and the neighbor individual direction. As people only see things in the front, $\phi_{\text {view }}$ controls how people see things. As shown in Figure. 6, the particle $P 1$ will have no influence on particle $P 6 . \omega_{i j}^{g}$ takes the particle group information into consideration, as shown in Figure. 6, the particle $P 1$ and particle $P 2$ are in the same group (cluster), they should have no influence on each other.

For a given scene or certain type of crowd with consistently similar sizes of objects we assume that $m_{i}=1$. Hence, we can simply estimate interaction force, $F_{\text {int }}$ from Eq. 4 for every particle as

$$
F_{\text {int }}=\frac{1}{\tau}\left(V_{i}^{p}-V_{i}\right)-\frac{d V_{i}}{d t}
$$




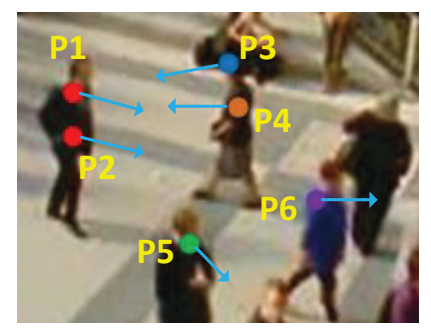

Fig. 6. Particles in a particular frame, with their current velocities. Color denotes interaction force values. Red color denotes high interaction force value, while green denotes a low value.

\section{Experiments}

Our algorithm is tested on three datasets: the publicly available dataset from University of Minnesota (UMN) ${ }^{1}$, PETS2009 ${ }^{2}$, and a web dataset [7]. Details are shown in the following subsections. In our experiments, all the video frames are resized to a fixed resolution with $480 \times 360$ pixels, and $3 D$ volumes of $5 \times 5 \times 10$ are adopted for constructing visual words. We compare our method with the optical flow based method(denoted as Optical Flow) and Social Force Model based method in [7] (denoted as SFM). In addition, for illustrating the effectiveness of particle group in interaction force computing, we conduct experiments using our approach without considering particle group information (denoted as Proposed-NC).

\subsection{The UMN Dataset}

This dataset comprises the videos of 11 different scenarios of an escape event. The videos are captured in 3 different indoor and outdoor scenes. Figure. 7(a) are some selected frames of these scenes. Each video clip starts with an initial part of normal behaviors and ends with sequences of abnormal behaviors. Scenes in this dataset are crowd, with about 20 people walking around.

We follow the same setup as in [7]. In particle advection phase, the resolution of the particle grid is kept at $25 \%$ of the number of original pixels. $K=30$ visual words are extracted from block of $T$ frames of force flow with one frame overlap. The STC is used to learn $L=30$ latent topics. The ROC curves in Figure. 7(b) demonstrate the performance of the proposed method outperforms these stateof-the-art methods in this dataset.

\subsection{The PETS2009 Dataset}

This section describes the results obtained on PETS 2009 'S3' dataset. This dataset is different from the above dataset, in the sense that abnormality begins

\footnotetext{
${ }^{1}$ mha.cs.umn.edu/movies/crowd-activity-all.avi

${ }^{2}$ http://ftp.cs.rdg.ac.uk/PETS2009
} 


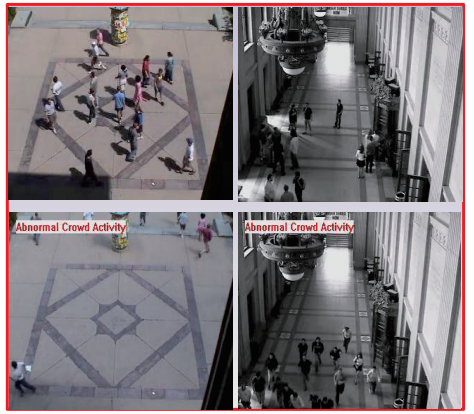

(a)

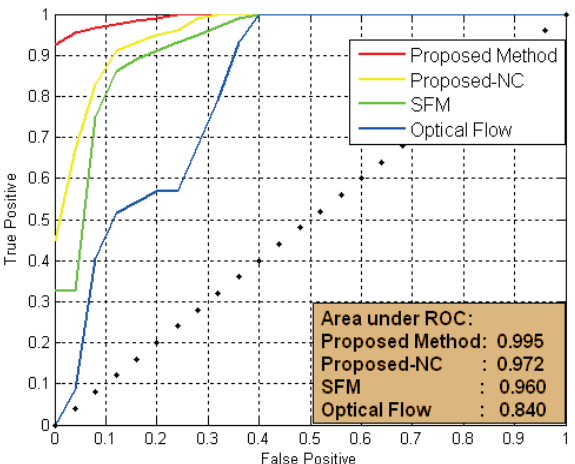

(b)

Fig. 7. (a) Top line: samples from normal events; Bottom line: samples from abnormal events. (b) The ROCs for abnormality detection on UMN dataset.

smoothly and this makes the detection more challenging, because of the gradual transition from normal to abnormal activity. Figure. 8(a) are some selected frames of these scenes.

In this experiment for all algorithms, the resolution of the particle grid in particle advection phase is kept at $30 \%$ of the number of original pixels. $K=30$ visual words are extracted from block of $T$ frames of force flow with one frame overlap. The STC is used to learn $L=40$ latent topics. The $R O C$ curves in Figure. 8(b) demonstrate the performance of the proposed method outperforms these state-of-the-art methods in this dataset.

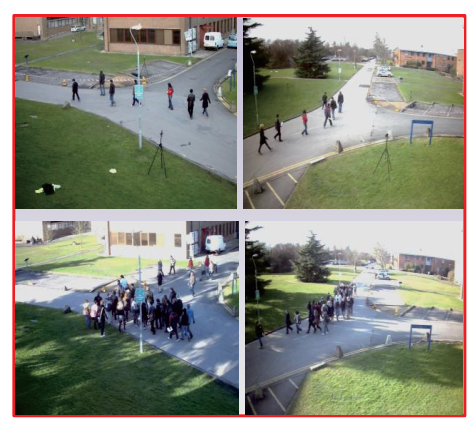

(a)

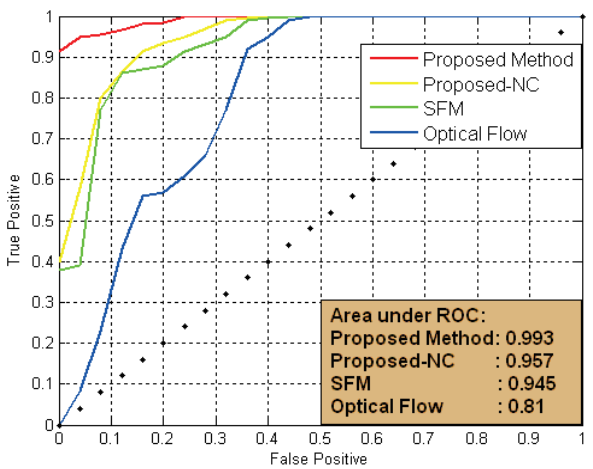

(b)

Fig. 8. (a) Top line: samples from normal events; Bottom line: samples from abnormal events. (b) The ROCs for abnormality detection on PETS 2009 'S3' dataset. 


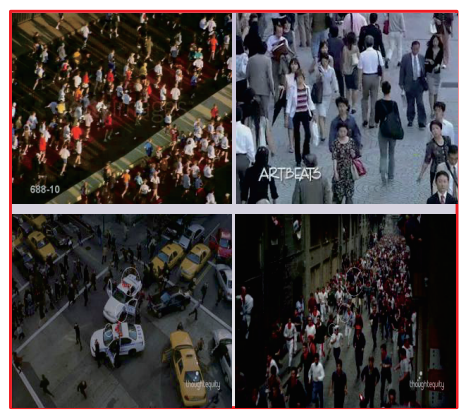

(a)

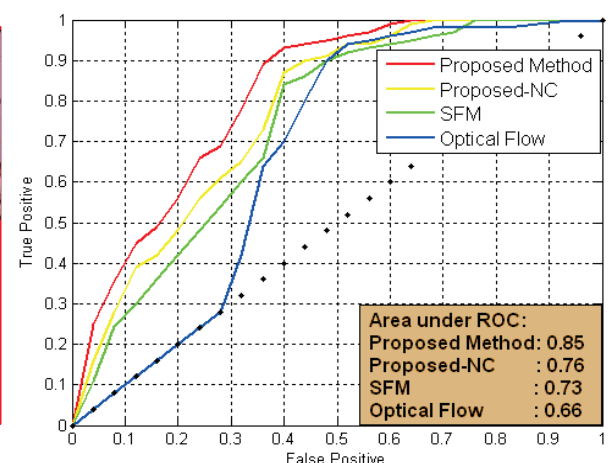

(b)

Fig. 9. (a)Top line: samples from normal events; Bottom line: samples from abnormal events. (b)The ROCs for abnormality detection on web dataset.

\subsection{The Web Dataset}

To evaluate the effectiveness of our algorithm, we also conduct an experiment on a more challenging dataset [7]. This dataset contains 12 sequences of normal crowd scenes, such as pedestrian walking, marathon running, and 8 scenes of abnormal scenes such as people fighting, escaping. Figure. 9(a) are some selected frames of these scenes.

We follow the same setup as in [7]. In particle advection phase, the resolution of the particle grid is kept at $10 \%$ of the number of original pixels. $K=30$ visual words are extracted from block of $T$ frames of force flow with one frame overlap. The STC is used to learn $L=50$ latent topics. In this experiment, our approach fails in classifying the scene of Marathon, as shown in Figure. 9(a). However, the performance of our approach outperforms these state-of-the-art methods, as shown in $R O C$ curves in Figure. 9(b).

\section{Conclusion}

In this paper, we propose a surrounding weighted interaction force estimation in the SFM-based framework. In our algorithm, the properties of surrounding individuals in terms of motion consistence, distance apart, and angle-of-view along moving directions are fully explored, in order to more precisely discriminate normal or abnormal behaviors of crowd. For computing the particle group information, a novel and high discriminative gait feature in frequency domain, combined with motion coherency and spatial proximity, is introduced in spectral clustering. Experimental results on three benchmarks demonstrate the effectiveness of our approach.

Acknowledgement. This work was supported by 973 ProgramProject No. 2010CB327905the National Natural Science Foundation of China (Grant No. 60903146, 60835002, 61070104). 


\section{References}

1. Helbing, D., Molnar, P.: Social force model for pedestrian dynamics. Physical Review E 51 (1995) 42-82

2. Sand, P., Teller, S.: Particle video: Long-range motion estimation using point trajectories. (2008) IJCV.

3. Zhu, J., Xing, E.: Sparse topical coding. In: UAI. (2011)

4. Chan, M., Hoogs, A., Schmiederer, J., Petersen, M.: Detecting rare events in video using semantic primitives with hmm. In: ICPR. (2004)

5. Dee, H., Hogg, D.: Detecting inexplicable behavior. In: BMVC. (2004)

6. Peter, H., Sebastian, T., Doretto, G., Krahnstoever, N., Rittscher, J., Yu, T.: Unified crowd segmentation. In: ECCV. (2008)

7. Mehran, R., Oyama, A., Shah, M.: Abnormal crowd behavior detection using social force model. In: CVPR. (2009)

8. Kratz, L., Nishino, K.: Anomaly detection in extremely crowded scenes using spatio-temporal motion pattern models. In: CVPR. (2009)

9. Yang, C., Yuan, J., Liu, J.: Sparse reconstruction cost for abnormal event detection. In: CVPR. (2011)

10. Wu, S., Moore, B., Shah, M.: Chaotic invariants of lagrangian particle trajectories for anomaly detection in crowded scenes. In: CVPR. (2010)

11. Ali, S., Sha, M.: A lgrangian particle dynamics approach for crowd flow segmentation and stability anysis. In: CVPR. (2007)

12. Mehran, R., Moore, B., Shah, M.: A streakline representation of flow in crowded scenes. In: ECCV. (2010)

13. Raghavendra, R., Alessio, D., Macro, C., Vittorio, M.: Optimizing interaction force for global anomaly detection in crowded scenes. In: ICCV Workshops. (2011)

14. Blei, D., Ng, A., Jordan, M.: Latent dirichlet allocation. JMLR 34 (1981) 993-1022

15. Lekien, F., Marsden, J.: Tricubic interpolation in three dimensions. Journal of Numerical Methods and Engineering 63 (2005) 455-471

16. Helbing, D., Buzna, L., Johansson, A., Werner, T.: Self-organized pedestrian crowd dynamics: Experiments, simulations and design solutions. Transportation Science 39 (2005) 1-24

17. Fu, W., Wang, J., Li, Z., Lu, H., Ma, S.: Learning semantic motion patterns for dynamic scenes by improved sparse topical coding. In: ICME. (2012)

18. Sochman, J., Hogg, D.: Who knows who - inverting the social force model for finding groups. In: ICCV Workshops. (2011)

19. Brostow, G., Cipolla, R.: Unsupervised bayesian detection of independent motion in crowds. In: CVPR. (2006)

20. Rabaud, V., Belongie, S.: Counting crowded moving objects. In: CVPR. (2006)

21. Makihara, Y., Sagawa, R., Mukaigawa, Y., Echigo, T., Yagi, Y.: Gait recognition using a view transformation model in the frequency domain. In: ECCV. (2006)

22. Lischinski, D.: Graphics gems iv, chapter incremental delaunay triangulation. (1994) Academic Press. 